\title{
Imaging reveals structural abnormalities in patients with GERD
}

The stomachs of patients with GERD have structural differences compared with healthy control individuals, according to new findings published in the American Journal of Gastroenterology.

"Most reflux events occur during spontaneous relaxations of the lower oesophageal sphincter; however, surprisingly, the number of these relaxations is not significantly different between healthy individuals and (most) patients with reflux disease," says author Mark Fox. "What it is that protects healthy individuals from reflux during relaxation of the reflux barrier is not known." Therefore, the researchers designed a study to help answer this question.

The team used MRI to visualize the soft tissue structures, whilst high resolution manometry was used to document oesophageal function in 24 healthy volunteers and 24 patients with GERD. These tests showed that the patients with
GERD had a wider insertion angle between the oesophagus and stomach than the healthy volunteers. The patients with GERD also had a larger opening of the oesophagogastric junction than the volunteers.

"This study also revealed other changes of oesophagogastric anatomy in reflux disease for the first time," says Fox. "These changes were not subtle. Most strikingly, the whole stomach appears to be rotated into an abnormal position within the abdomen." Fox and his team hope that their findings will aid the development of new and better surgical treatment of GERD that restores the normal structure and function of the reflux barrier.

\section{Claire Greenhill}

Original article Curcic, J. et al. Abnormal structure and function of the esophagogastric junction and proximal stomach in gastroesophageal reflux disease. Am. J. Gastroenterol. doi:10.1038/ajg.2014.25 